RIMS-1728

A Note on Polylinking Flow Networks

By

Satoru FUJISHIGE

$\underline{\text { June } 2011}$

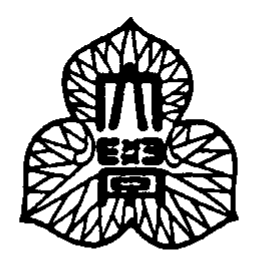

京都大学 数理解析研究所

RESEARCH INSTITUTE FOR MATHEMATICAL SCIENCES

KYOTO UNIVERSITY, Kyoto, Japan 


\title{
A Note on Polylinking Flow Networks
}

\author{
SATORU FUJISHIGE \\ Research Institute for Mathematical Sciences \\ Kyoto University, Kyoto 606-8502, Japan \\ fujishig@kurims.kyoto-u.ac.jp
}

June 30, 2011

\begin{abstract}
This is a supplementary note on M. X. Goemans, S. Iwata, and R. Zenklusen's paper that proposes a flow model based on polylinking systems. Their flow model is a series (or tandem) connection of polylinking systems. We can consider an apparently more general model of a polylinking flow network which consists of an ordinary arccapacitated network endowed with polylinking systems on the vertex set, one for each vertex of the network. This is a natural, apparent generalization of polymatroidal flow model of E. L. Lawler and C. U. Martel and of generalized-polymatroidal flow model of R. Hassin. We give a max-flow min-cut formula for the polylinking network flow problem and discuss some acyclic flow property of polylinking flows.
\end{abstract}

Keywords: Linking systems, Polylinking flows, Submodular functions

Mathematics Subject Classification (2000): 90C27, 90B10, 90C90

\section{Introduction}

M. X. Goemans, S. Iwata, and R. Zenklusen [6] proposed a flow model based on polylinking systems of A. Schrijver [9]. The present note is supplementary to their paper and points out an apparent generalization of their model, which is also a natural, apparent generalization of polymatroidal flow model of E. L. Lawler and C. U. Martel [8] and of generalizedpolymatroidal flow model of R. Hassin [7]. We give a max-flow min-cut formula for the polylinking network flow problem and discuss some acyclic flow property of polylinking flows. The results are easy consequences of those in the theory of submodular functions but it may be worth noting and useful for wireless information networks [1], which motivated the work of [6] 


\section{Preliminaries: Base polyhedra and polylinking systems}

Let $W$ be a nonempty set and $f: 2^{W} \rightarrow \mathbf{R}$ be a submodular function, i.e., $f$ satisfies

$$
f(X)+f(Y) \geq f(X \cup Y)+f(X \cap Y) \quad(\forall X, Y \subseteq W) .
$$

We assume $f(\emptyset)=0$. The base polyhedron associated with $f$ is defined by

$$
\mathrm{B}(f)=\left\{x \in \mathbf{R}^{W} \mid \forall X \subseteq W: x(X) \leq f(X), x(W)=f(W)\right\} .
$$

Here for simplicity we consider submodular functions on power sets (or Boolean lattices) but we can easily adapt the arguments in this note to submodular functions on ring families (or distributive lattices). A vector $x \in \mathrm{B}(f)$ is called a base. For any base $x \in \mathrm{B}(f)$ and $u \in W$ we define $\operatorname{dep}(x, u)$ by

$$
\operatorname{dep}(x, u)=\left\{v \in W \mid \exists \alpha>0: x+\alpha\left(\chi_{u}-\chi_{v}\right) \in \mathrm{B}(f)\right\},
$$

where for any $w \in W \chi_{w}$ is the unit vector such that $\chi_{w}(w)=1$ and $\chi_{w}(s)=0$ for all $s \in W \backslash\{w\}$. In other words, when $v \in \operatorname{dep}(x, u) \backslash\{u\}$, we can increase $x(u)$ and at the same time decrease $x(v)$ by some positive amount without leaving the base polyhedron $\mathrm{B}(f)$. The function dep : $\mathrm{B}(f) \times W \rightarrow 2^{W}$ is called the dependence function. Moreover, for any $v \in \operatorname{dep}(x, u) \backslash\{u\}$ define

$$
\tilde{\mathrm{c}}(x, u, v)=\max \left\{\alpha \in \mathbf{R} \mid x+\alpha\left(\chi_{u}-\chi_{v}\right) \in \mathrm{B}(f)\right\},
$$

which is called the exchange capacity from $v$ to $u$ associated with base $x$. Dependence functions and exchange capacities will appear only in Section 4.3. For more details about the theory of submodular functions see [5].

For any vector $x \in \mathbf{R}^{W}$ and any subset $U$ of $W$ define $x^{U}$ to be the vector in $\mathbf{R}^{U}$ such that $x^{U}(u)=x(u)$ for all $u \in U$, which is the restriction of $x$ to $U$. For any disjoint nonempty subsets $U_{1}, U_{2} \subset W$ and any vectors $x \in \mathbf{R}^{U_{1}}$ and $y \in \mathbf{R}^{U_{2}}$ denote by $x \oplus y$ the vector in $\mathbf{R}^{U_{1} \cup U_{2}}$ such that $(x \oplus y)(u)=x(u)$ for all $u \in U_{1}$ and $(x \oplus y)(u)=y(u)$ for all $u \in U_{2}$.

Suppose that $f(W)=0$ and $f(X) \geq 0$ for all $X \subseteq W$, which implies $\mathbf{0} \in \mathrm{B}(f)$. We assume this property for all submodular functions appearing in the sequel. Let $\left(U_{1}, U_{2}\right)$ be an ordered pair of nonempty subsets of $W$ such that $U_{1} \cap U_{2}=\emptyset$ and $U_{1} \cup U_{2}=W$. We call it an ordered proper bisection of $W$. Consider a reflection by $U_{1}$ of the base polyhedron given by

$$
\mathrm{B}_{\left(U_{1}, U_{2}\right)}(f)=\left\{y \mid x \in \mathrm{B}(f), y^{U_{1}}=-x^{U_{1}}, y^{U_{2}}=x^{U_{2}}\right\} .
$$

Then the triple $\left(U_{1}, U_{2}, \mathrm{~B}_{\left(U_{1}, U_{2}\right)}(f)\right)$ is a polylinking system and $\mathrm{B}_{\left(U_{1}, U_{2}\right)}(f)$ is the associated polylinking polyhedron. We call $f$ the submodular function associated with the polylinking system. Here we define a polylinking system by means of a submodular function (cf. [5, Sec. 3.5(b)]). (The original polylinking system introduced by Schrijver [9] considers the restriction of $\mathrm{B}_{\left(U_{1}, U_{2}\right)}(f)$ to the nonnegative orthant $\mathbf{R}_{+}^{W}$.) For any $y \in \mathrm{B}_{\left(U_{1}, U_{2}\right)}(f)$ we say $y^{U_{1}}$ is linked to $y^{U_{2}}$, and $\left(y^{U_{1}}, y^{U_{2}}\right)$ is called a pair of linked vectors. Note that $y\left(U_{1}\right)=y\left(U_{2}\right)$ since $f(W)=0$ by definition. 


\section{The Polylinking Flow Model of Goemans, Iwata, and Zenklusen}

Now let us give a description of the polylinking flow model of Goemans, Iwata, and Zenklusen [6] for completeness of the presentation. Consider nonempty disjoint sets $V_{i}(i=$ $1, \cdots, r)$ with an integer $r \geq 2$ and polylinking systems $\left(V_{1}, V_{i+1}, L_{i}\right)(i=1, \cdots, r-1)$. The pair $(V, L)$, where $V=\left(V_{1}, \cdots, V_{r}\right)$ and $L=\left(L_{1}, \cdots, L_{r-1}\right)$, is called a polylinking flow model in [6]. It is a series (or tandem) connection of polylinking systems. A flow in the polylinking flow model $(V, L)$ is a tuple $x=\left(x_{1}, \cdots, x_{r}\right)$ such that $\left(x_{i}, x_{i+1}\right)$ is a pair of linked vectors in $L_{i}$ for all $i=1, \cdots, r-1$ and $x_{i}$ is nonnegative for all $i=1, \cdots, r$. Note that we always have a feasible flow consisting of zero linked vectors. We have a common value $x_{1}\left(V_{1}\right)=\cdots=x_{r}\left(V_{r}\right)$, which is called the value of flow $x=\left(x_{1}, \cdots, x_{r}\right)$.

Goemans, Iwata, and Zenklusen [6] considered a problem of finding a flow of maximum value in the polylinking flow model, showed a min-max formula, and gave an efficient algorithm for finding a maximum flow in the polylinking flow model by reducing the problem to a submodular flow problem of J. Edmonds and R. Giles [2] and by employing an efficient algorithm for submodular flows such as L. Fleischer and Iwata's [3] together with the fast Fourier transformation on finite fields.

\section{Polylinking Flow Networks}

Goemans, Iwata, and Zenklusen [6] considered a series (or tandem) connection of polylinking systems. We can consider an apparently more general model which consists of an ordinary arc-capacitated network endowed with polylinking systems, one for each vertex of the network. This is a natural, apparent generalization of a polymatroidal flow model of Lawler and Martel [8] and that of a generalized-polymatroidal flow of Hassin [7].

\subsection{Definition of a (general) polylinking flow network}

Let $G=(V, A)$ be a directed graph with a vertex set $V$ and an arc set $A$, and let $\underline{c}: A \rightarrow$ $\mathbf{R} \cup\{-\infty\}$ and $\bar{c}: A \rightarrow \mathbf{R} \cup\{+\infty\}$ be lower and upper capacity functions on arc set $A$ such that $\underline{c}(a) \leq \bar{c}(a)$ for all $a \in A$. For each vertex $v \in V$ we are given a linking system $\left(\delta^{-} v, \delta^{+} v, L_{v}\right)$, where let $f_{v}: 2^{\delta^{-} v \cup \delta^{+} v} \rightarrow \mathbf{R}$ be the submodular function associated with the linking polyhedron $L_{v}$. (For any vertex $v \in V, \delta^{-} v$ denotes the set of arcs in $G$ whose terminal vertices are $v$, and $\delta^{+} v$ the set of arcs in $G$ whose initial vertices are $v$.) We call $\mathcal{N}=(G, c, \boldsymbol{L})$ a polylinking flow network, where $\boldsymbol{L}=\left(L_{v} \mid v \in V\right)$.

A feasible flow (or a polylinking flow) in the polylinking network $\mathcal{N}=(G, c, \boldsymbol{L})$ is a function $\varphi: A \rightarrow \mathbf{R}$ that satisfies the following.

$$
\underline{c}(a) \leq \varphi(a) \leq \bar{c}(a) \quad(\forall a \in A)
$$




$$
\varphi^{\delta^{-} v \cup \delta^{+} v} \in L_{v} \quad(\forall v \in V),
$$

where recall that $\varphi^{F}$ for $F \subseteq A$ is the restriction of $\varphi$ to $F$.

Remark 1: A polymatroidal flow network of Lawler and Martel [8] is a special case of a linking flow network where each linking polyhedron $L_{v}$ for $v \in V$ is a composition of polymatroids on $\delta^{-} v$ and on $\delta^{+} v$, which is defined as follows. For two polymatroid polyhedra $P_{1} \subset \mathbf{R}^{S_{1}}$ and $P_{2} \subset \mathbf{R}^{S_{2}}$ with $S_{1} \cap S_{2}=\emptyset$ define a polytope $L\left(P_{1}, P_{2}\right) \subset \mathbf{R}^{S_{1} \cup S_{2}}$ by

$$
L\left(P_{1}, P_{2}\right)=\left\{x_{1} \oplus x_{2} \mid x_{1} \in P_{1}, x_{2} \in P_{2}, x_{1}\left(S_{1}\right)=x_{2}\left(S_{2}\right)\right\} .
$$

We can see that the reflection of $L\left(P_{1}, P_{2}\right)$ by $S_{1}$ is a base polyhedron, and hence $L\left(P_{1}, P_{2}\right)$ gives a polylinking polyhedron. Also Hassin [7] considered a linking flow network when each linking polyhedron $L_{v}$ is a composition of generalized polymatroids [4] on $\delta^{-} v$ and on $\delta^{+} v$ for $v \in V$, which is defined similarly as above by replacing polymatroids by generalized polymatroids.

These facts can be understood as follows. Let $P_{1} \subset \mathbf{R}^{S_{1}}$ and $P_{2} \subset \mathbf{R}^{S_{2}}$ be generalized polymatroids. We embed $P_{1}$ (resp. $P_{2}$ ) in $\mathbf{R}^{S_{1} \cup S_{2}}$ by taking the direct sum of $P_{1}$ (resp. $P_{2}$ ) with the zero vector in $\mathbf{R}^{S_{2}}$ (resp. $\mathbf{R}^{S_{1}}$ ). Then consider a new element $e_{0}$ commonly used for $P_{1}$ and $P_{2}$, put $T=S_{1} \cup S_{2} \cup\left\{e_{0}\right\}$, and let $B_{1} \subset \mathbf{R}^{T}$ and $B_{2} \subset \mathbf{R}^{T}$ be, respectively, the base polyhedra lying in the hyperplane $x(T)=0$ such that the projection along the axis $e_{0}$ into the hyperplane $x\left(e_{0}\right)=0$ are $P_{1}$ and $P_{2}$ (after being restricted on $S_{1}$ and $S_{2}$ ) [5, Sec. 3.5(a)]. Then, the Minkowski sum of $-B_{1}$ and $B_{2}$ is a base polyhedron, denoted by $B_{1,2}$, in $\mathbf{R}^{T}$, where $-B_{1}=\left\{-x \mid x \in B_{1}\right\}$ is also a base polyhedron. Taking a section of $B_{1,2}$ by the hyperplane $x\left(e_{0}\right)=0$ and restricting it to $T \backslash\left\{e_{0}\right\}=S_{1} \cup S_{2}$, we get a base polyhedron $\hat{B}$ in $\mathbf{R}^{S_{1} \cup S_{2}}$. Finally, by the reflection of $\hat{B}$ by $S_{1}$ we obtain the linking polyhedron $L\left(P_{1}, P_{2}\right)$ defined by (4.3) for generalized polymatroids $P_{1} \subset \mathbf{R}^{S_{1}}$ and $P_{2} \subset \mathbf{R}^{S_{2}}$.

\subsection{Equivalence between polylinking flows and submodular flows}

Now we show that any polylinking flow network can be reduced to a submodular flow network. The reduction technique given below is the same as the one shown in [5, Sec. 5.2(c)] though polymatroids are considered instead of polylinking systems.

Given a graph $G=(V, A)$, lower and upper capacity functions $\underline{c}: A \rightarrow \mathbf{R}$ and $\bar{c}:$ $A \rightarrow \mathbf{R}$ with $\underline{c}(a) \leq \bar{c}(a)$ for all $a \in A$, and a submodular function $f: 2^{V} \rightarrow \mathbf{R}$ with $f(\emptyset)=f(V)=0$, a submodular flow is a function $\varphi: A \rightarrow \mathbf{R}$ that satisfies

$$
\begin{aligned}
& \underline{c}(a) \leq \varphi(a) \leq \bar{c}(a) \quad(\forall a \in A), \\
& \partial \varphi \in \mathrm{B}(f),
\end{aligned}
$$

where $\partial \varphi$ is the boundary of flow $\varphi$ defined by $\partial \varphi(v)=\sum_{a \in \delta^{+}{ }_{v}} \varphi(a)-\sum_{a \in \delta^{-} v} \varphi(a)$ for all $v \in V$. 
For any polylinking flow network $\mathcal{N}=\left(G=(V, A), \underline{c}, \bar{c}, \boldsymbol{L}=\left(L_{v} \mid v \in V\right)\right)$ with associated submodular functions $f_{v}: 2^{\delta^{-} v \cup \delta^{+} v} \rightarrow \mathbf{R}$ for all $v \in V$, construct a submodular flow network $\mathcal{N}_{0}=\left(G_{0}=\left(V_{0}, A\right), \underline{c}, \bar{c}, f_{0}\right)$ as follows.

$$
\begin{aligned}
& V_{0}=\bigcup_{v \in V}\left(W_{v}^{-} \cup W_{v}^{+}\right), \\
& \quad W_{v}^{-}=\left\{u_{a}^{-} \mid a \in \delta^{-} v\right\}, \quad W_{v}^{+}=\left\{u_{a}^{+} \mid a \in \delta^{+} v\right\} \quad(\forall v \in V), \\
& f(U)=\sum_{v \in V} \bar{f}_{v}\left(\left(W_{v}^{-} \cup W_{v}^{+}\right) \cap U\right) \quad\left(\forall U \subseteq V_{0}\right),
\end{aligned}
$$

where $\bar{f}_{v}$ is the submodular function on $2^{W_{v}^{-} \cup W_{v}^{+}}$that is identified with $f_{v}$ by the natural correspondence between $\delta^{-} v \cup \delta^{+} v$ and $W_{v}^{-} \cup W_{v}^{+}$. It is easy to see that $\varphi: A \rightarrow \mathbf{R}$ is a polylinking flow in $\mathcal{N}$ if and only if $\varphi$ is a submodular flow in $\mathcal{N}_{0}$.

Remark 2: Similarly as in [5, Sec. 5.2] we can show that any submodular flow network can be reduced to a polylinking flow network. Hence these two models are equivalent. That is, the polylinking flow problem is what is called a neoflow problem in [5, Sec. 5]. From now on we consider both networks $\mathcal{N}$ and $\mathcal{N}_{0}$ and identify a polylinking flow $\varphi$ in $\mathcal{N}$ with its corresponding submodular flow $\varphi$ in $\mathcal{N}_{0}$. Note that the two flows are the same function on $A$.

Suppose that we are given a reference arc $a_{0} \in A$. Then we have a max-flow min-cut theorem as follows (see [5, Theorem 5.11]).

Theorem 4.1: Suppose that there exists a feasible linking flow in $\mathcal{N}$ (or equivalently a feasible submodular flow in $\mathcal{N}_{0}$ ). Then we have

$$
\begin{aligned}
& \max \left\{\varphi\left(a_{0}\right) \mid \varphi: \text { a feasible linking flow in } \mathcal{N}\right\} \\
& =\min \left\{\bar{c}\left(a_{0}\right), \min \left\{\bar{c}\left(\Delta^{+} X\right)-\underline{c}\left(\Delta^{-} X \backslash\left\{a_{0}\right\}\right)+f(V \backslash X) \mid X \subseteq V_{0}, a_{0} \in \Delta^{-} X\right\}\right\},
\end{aligned}
$$

where operators $\Delta^{ \pm}$appearing in the right-hand side are defined with respect to graph $G_{0}=\left(V_{0}, A\right)$ for network $\mathcal{N}_{0}\left(\Delta^{+} X\right.$ is the set of arcs leaving $X$ and $\Delta^{-} X$ the set of arcs entering $X)$. Moreover, if $\underline{c}, \bar{c}$, and $f$ are integer-valued, then there exists an integral maximum linking flow in $\mathcal{N}$ (with respect to reference arc $\left.a_{0}\right)$.

\subsection{Existence of acyclic polylinking flows of given flow value}

It is well-known that for any two-terminal flow $\varphi$ in a classical flow network there exists a two-terminal flow $\psi$ such that the two flow values are the same, flow $\psi$ is $\varphi$-equisignum (i.e. $\psi(a)>0$ implies $\varphi(a)>0$ and $\psi(a)<0$ implies $\varphi(a)<0$ ), and the network restricted on the support of $\psi$ is acyclic. We consider such a property for polylinking flows. 
For simplicity let us assume $\underline{c}(a)=0$ for all $a \in A$. Let $\varphi$ be a feasible flow in network $\mathcal{N}_{0}$. Define an auxilliary graph $G_{\varphi}=\left(V_{0}, A_{\varphi}\right)$ as follows.

$$
\begin{aligned}
& A_{\varphi}= A_{\varphi}^{+} \cup \bigcup_{v \in V} D_{\varphi}^{v}, \\
& A_{\varphi}^{+}=\{a \mid a \in A, \varphi(a)>0\}, \\
& D_{\varphi}^{v}=\left\{\left(u_{a}^{-}, u_{b}^{+}\right) \mid a \in \delta^{-} v, b \in \delta^{+} v, b \in \operatorname{dep}_{v}\left(\left(-\varphi^{\delta^{-}} v\right) \oplus \varphi^{\delta^{+} v}, a\right)\right\} \\
& \quad(\forall v \in V),
\end{aligned}
$$

where $\delta^{ \pm}$are those defined with respect to graph $G=(V, A)$ for network $\mathcal{N}$ and $\operatorname{dep}_{v}$ for $v \in V$ is the dependence function associated with $f_{v}$ and a base $\left(-\varphi^{\delta^{-} v}\right) \oplus \varphi^{\delta^{+} v} \in \mathrm{B}\left(f_{v}\right)$. (Recall that $b \in \operatorname{dep}_{v}\left(\left(-\varphi^{\delta^{-} v}\right) \oplus \varphi^{\delta^{+} v}, a\right)$ means that we can decrease $\varphi(a)$ and $\varphi(b)$ by some (and the same) amount $\alpha>0$ while keeping $\varphi^{\delta^{-} v \cup \delta^{+} v} \in L_{v}$. The maximum of such values $\alpha$ is called the exchange capacity from $b$ to $a$ with respect to base $\left(-\varphi^{\delta^{-} v}\right) \oplus \varphi^{\delta^{+} v} \in \mathrm{B}\left(f_{v}\right)$ and is denoted by $\tilde{\mathrm{c}}_{v}\left(\left(-\varphi^{\delta^{-} v}\right) \oplus \varphi^{\delta^{+} v}, a, b\right)$.)

The following algorithmic property is well-known [5, Sec. 5.5].

- If there exists a directed cycle in the auxiliary graph $G_{\varphi}$, let $Q$ be one of such directed cycles, regarded as a subset of $A_{\varphi}$, that do not have any short-cuts. Then we can obtain a new feasible flow $\varphi^{\prime}$ by

$$
\varphi^{\prime}(a)= \begin{cases}\varphi(a)-\alpha & (a \in Q) \\ \varphi(a) & (a \in A \backslash Q),\end{cases}
$$

where $\alpha$ is a positive number less than or equal to

$$
\begin{aligned}
& \min \left\{\min \left\{\varphi(a) \mid a \in Q \cap A_{\varphi}^{+}\right\},\right. \\
& \left.\quad \min \left\{\tilde{\mathrm{c}}_{v}\left(\left(-\varphi^{\delta^{-} v}\right) \oplus \varphi^{\delta^{+} v}, u_{a}^{-}, u_{b}^{+}\right) \mid\left(u_{a}^{-}, u_{b}^{+}\right) \in Q \cap D_{\varphi}^{v}, v \in V\right\}\right\} .
\end{aligned}
$$

Hence, given a feasible flow $\varphi$ in $\mathcal{N}_{0}$, reducing flows along directed cycles not containing reference arc $a_{0}$, we can obtain a feasible flow $\psi$ of the same flow value as $\varphi$ such that $\psi$ is $\varphi$-equisignum and the auxiliary graph $G_{\psi}$ has no directed cycle $Q$ with $a_{0} \notin Q$.

We have the following acyclic reduction property of polylinking flows in general polylinking networks.

Theorem 4.2: Given a feasible flow $\varphi$ in polylinking network $\mathcal{N}$ with a reference arc $a_{0}$ and $\underline{c}=0$, there exists a feasible flow $\psi$ that has the same flow value as $\varphi$ and is $\varphi$-equisignum and there exists no directed cycle $Q$ with $a_{0} \notin Q$ in the auxiliary graph $G_{\psi}$.

(Proof) Re-define

$$
\begin{aligned}
& \bar{c}(a)=\varphi(a) \quad(\forall a \in A), \\
& \underline{c}\left(a_{0}\right)=\varphi\left(a_{0}\right) .
\end{aligned}
$$


Moreover, consider a cost function $\gamma: A \rightarrow \mathbf{R}$ such that $\gamma(a)=1$ for all $a \in A$. Then let $\psi$ be the minimum-cost submodular flow in $\mathcal{N}_{0}$ with the upper and lower capacity functions and the cost function defined as above. The optimality of $\psi$ [5, Sec. 5.4] implies that there does not exist any directed cycle in the auxiliary graph $G_{\psi}$ for the re-defined network $\mathcal{N}_{0}$. It follows that the flow $\psi$ satisfies the condition of the statement in the present theorem.

Remark 3: The wireless information network model (ADT-network) considered in [1] is a two-terminal acyclic network with a source-sink pair. Practical wireless networks usually contain directed cycles and source-sink pairs may change from time to time. Theorem 4.1 shows a max-flow min-cut theorem for ADT-networks with possible directed cycles. Theorem 4.2 further shows that for a given source-sink pair $(s, t)$ there exists a maximum information flow $\psi$ from $s$ to $t$ such that $G_{\psi}$ is acyclic, so that the flow can be realized as a flow in an ADT-network. Here it should be noted that arcs in $\{a \mid \psi(a)>0\}$ may form a directed cycle in $G$ of the polylinking network $\mathcal{N}$ but the information flow is not rejoined at any vertex (in $G_{\psi}$ ) which it left before, since $G_{\psi}$ is acyclic. Such an acyclic flow can be obtained by the use of any existing algorithms for (minimum-cost) submodular flows.

\section{Acknowledgments}

The author is grateful to Satoru Iwata for his useful comments on an earlier version of this note.

\section{References}

[1] A. S. Avestimehr, S. N. Diggavi, and D. N. C. Tse: Wireless network information flow: a deterministic approach. Proceedings of the Allerton Conference on Communication, Control, and Computing (2007); IEEE Transactions on Information Theory (to appear).

[2] J. Edmonds and R. Giles: A min-max relation for submodular functions on graphs. Annals of Discrete Mathematics 1 (1977) 185-204.

[3] L. Fleischer and S. Iwata: Improved algorithms for submodular function minimization and submodular flow. In: Proceedings of the 32nd ACM Symposium on Theory of Computing (2000), pp. 107-116.

[4] A. Frank: Generalized polymatroids. In: Finite and Infinite Sets, I (A. Hajnal, L. Lovász, and V. T. Sós, eds., Colloquia Mathematica Societatis János Bolyai 37, NorthHolland, Amsterdam, 1984), pp. 285-294. 
[5] S. Fujishige: Submodular Functions and Optimization (Annals of Discrete Mathematics 47, North-Holland, 1991), and Second Edition (Annals of Discrete Mathematics 58, Elsevier, 2005).

[6] M. X. Goemans, S. Iwata, and R. Zenklusen: A flow model based on polylinking systems. Mathematical Programming, Ser. A (to appear) (published online: 05 March 2011).

[7] R. Hassin: Minimum-cost flow with set constraints. Networks 12 (1982) 1-21.

[8] E. L. Lawler and C. U. Martel: Computing maximal polymatroidal network flows. Mathematics of Operations Research 7 (1982) 334-347.

[9] A. Schrijver: Matroids and Linking Systems (Mathematical Centre Tracts 88, Amsterdam, 1978). 